\title{
Multidimensional latent trait linear mixed model: an application in clinical studies with multivariate longitudinal outcomes
}

\author{
Jue Wang and Sheng Luo*广†
}

\begin{abstract}
Multilevel item response theory (MLIRT) models have been widely used to analyze the multivariate longitudinal data of mixed types (e.g., categorical and continuous) in clinical studies. The MLIRT models often have unidimensional assumption, that is, the multiple outcomes are clinical manifestations of a univariate latent variable. However, the unidimensional assumption may be unrealistic because some diseases may be heterogeneous and characterized by multiple impaired domains with variable clinical symptoms and disease progressions. We relax this assumption and propose a multidimensional latent trait linear mixed model (MLTLMM) to allow multiple latent variables and within-item multidimensionality (one outcome can be a manifestation of more than one latent variable). We conduct extensive simulation studies to assess the unidimensional MLIRT model and the proposed MLTLMM model. The simulation studies suggest that the MLTLMM model outperforms unidimensional model when the multivariate longitudinal outcomes are manifested by multiple latent variables. The proposed model is applied to two motivating studies of amyotrophic lateral sclerosis: a clinical trial of ceftriaxone and the Pooled Resource Open-Access ALS Clinical Trials database. Copyright @ 2017 John Wiley \& Sons, Ltd.
\end{abstract}

Keywords: clinical trial; item response theory; latent variable model; MCMC; random effects

\section{Introduction}

Amyotrophic lateral sclerosis (ALS) is a neurodegenerative disease characterized by progressive muscular paralysis reflecting degeneration of motor neurones in the primary motor cortex, corticospinal tracts, brainstem, and spinal cord [1]. ALS is the most common motor neuron disease and fatal with median survival of 3 years from diagnosis. Currently, the pathogenesis of ALS is unknown, and there is no cure. Many clinical trials are conducted to search for neuroprotective treatments capable of extending the length and meaningful quality of life for patients (e.g., the study of ceftriaxone [2], the Pooled Resource Open-Access ALS Clinical Trials (PRO-ACT) database [3], and the study of MCI-186 [4]). ALS causes impairment in multiple domains (e.g., bulbar, fine motor, and gross motor). The disease progresses heterogeneously in time and across domains and individuals: The trajectory of progression may vary between different domains, both within and across ALS patients. Therefore, ALS studies repeatedly collect multiple outcomes of mixed types (categorical and continuous) to obtain the full spectrum of the disease severity and progression. Thus, data have a natural multivariate longitudinal structure.

We illustrate the complexity of the data generated by the ALS studies. The ALS Functional Rating Scale (ALSFRS) is frequently used to evaluate patients' overall disease severity [5-7], and it is an instrument for evaluating multiple clinical aspects of ALS, including bulbar, fine motor, gross motor function, and respiratory disability [8]. The ALSFRS consists of 10 ordinal items (Table S1) rating level of functional impairment in performing common tasks, for example, speech (item 1), and cutting food (item 5). Each item is rated on a 5-point scale from 0 (unable to attempt the task) to 4 (normal ability). Ten items are often summed up to obtain the ALSFRS total score, which is treated as a continuous variable and is analyzed using a regression model under normality assumption. However, this analysis based on

Department of Biostatistics, The University of Texas Health Science Center at Houston, Houston, TX 77030, U.S.A.

${ }^{*}$ Correspondence to: Sheng Luo, Department of Biostatistics, The University of Texas Health Science Center at Houston, 1200

Pressler St, Houston, TX 77030, U.S.A.

${ }^{\dagger}$ E-mail: sheng.t.luo@uth.tmc.edu 
a sum-score is problematic because the total score is discrete, asymmetric with ceiling or floor effects, and items have varying sensitivity to change (refer to as curvilinearity, e.g., it may take, on average, 24 and 12 months to have one point drop in items of speech and cutting food, respectively) $[9,10]$. Moreover, the sum-score analysis leads to a loss of information because the sum score ignores the differences between response patterns leading to the same sum score [11].

Alternatively, one can analyze the longitudinal scores on the individual items using a multilevel item response theory (MLIRT) model [12-15]. Specifically, the multiple items are viewed as clinical manifestations of an unobserved true disease severity represented by a univariate latent variable (LV; unidimensional assumption), which is regressed on predictors (e.g., treatment and time) and subject-specific random effects (describing the between-subject differences). Some extensions of the MLIRT models have been developed. For example, Verhagen and Fox [16] extended the MLIRT model by accounting for item parameter shift (item-specific parameters change during the study). Schmidt et al [17] proposed a pretestposttest-posttest multivariate MLIRT model for repeated measures. Although the MLIRT model directly analyzes the raw item scores and addresses the aforementioned issues of analyzing the sum-score, the unidimensional assumption may be unrealistic in the study of ALS because it is a heterogeneous disorder characterized by multiple impaired domains (e.g., bulbar, fine motor, and gross motor functions) with variable disease progression [18] and some items may cross load on multiple domains.

To this end, te Marvelde et al [19], Cho and Cohen [20], and Cho et al [21] developed multidimensional (multiple LVs) longitudinal item response theory models for binary response variables. However, in their models, the means of LVs and variances and covariances among LVs do not depend on any individual information. In general, a multidimensional item response theory model makes it possible to investigate correlations between LVs, to test simultaneously effects of an explanatory variable on several LVs and to test for differential effects of explanatory variables on various LVs [22]. To address this issue, Fox et al [22] developed a multidimensional item response model with randomized response techniques for cross-sectional data. Klein Entink et al [23] and Fox and Marianti [24] jointly modeled the accuracy and speed of test takers using two MLIRT models (one for accuracy and one for speed). However, the current state-of-the-art LV models do not simultaneously incorporate multiple LVs and within-item multidimensionality (one outcome can be a manifestation of more than one LV) to analyze the longitudinal data.

In this article, we propose a multidimensional latent trait linear mixed model (MLTLMM) that allows multiple LVs and within-item multidimensionality. The MLTLMM model has two levels. The first level multidimensional latent trait model quantifies the relationship between a patient's multiple latent disease severity scores and the observed multivariate outcomes (e.g., 10 items in the ALSFRS), while the secondlevel linear mixed model connects the latent disease scores to observed covariates and subject-specific random effects. Because the number of latent disease scores is much smaller than the number of observed outcomes, the MLTLMM model can be used with a large number of outcomes and is more computational scalable than multivariate marginal and random effects models. Additional advantages include (i) easy handling of unbalanced data and outcomes of mixed types; (ii) explicit accounting for correlation structures using random effects; and (iii) seamless incorporation of fixed and random effects, as well as interactions of covariates with time (which can not be easily accommodated in structural equation models [25, 26], as discussed in An et al. [27]).

The rest of the article proceeds as follows. In Section 2, we describe two motivating ALS studies and the data structure. Section 3 discusses the proposed MLTLMM model, Bayesian inference, and model selection criteria. Section 4 provides an extensive simulation study to assess the performance of the MLTLMM model. In Section 5, we apply the MLTLMM model to the motivating studies. Section 6 gives some concluding remarks and discussions.

\section{Motivating clinical studies}

The methodological development is motivated by two ALS clinical studies, including the ceftriaxone study [2]; a double-blind, placebo controlled multi-phase clinical trial with 513 subjects to determine if ceftriaxone slows the disease progression of ALS; and the PRO-ACT database [3], which combines fully de-identified records of participants from 23 ALS clinical trials. In 2012, a computational challenge was ran by Prize4Life and The DREAM Project utilizing data from the PRO-ACT database to promote ALS research. In this article, we use the data for the challenge, which is a subset of the PRO-ACT database, including 918 participants (202 in placebo and 716 in treatment). 

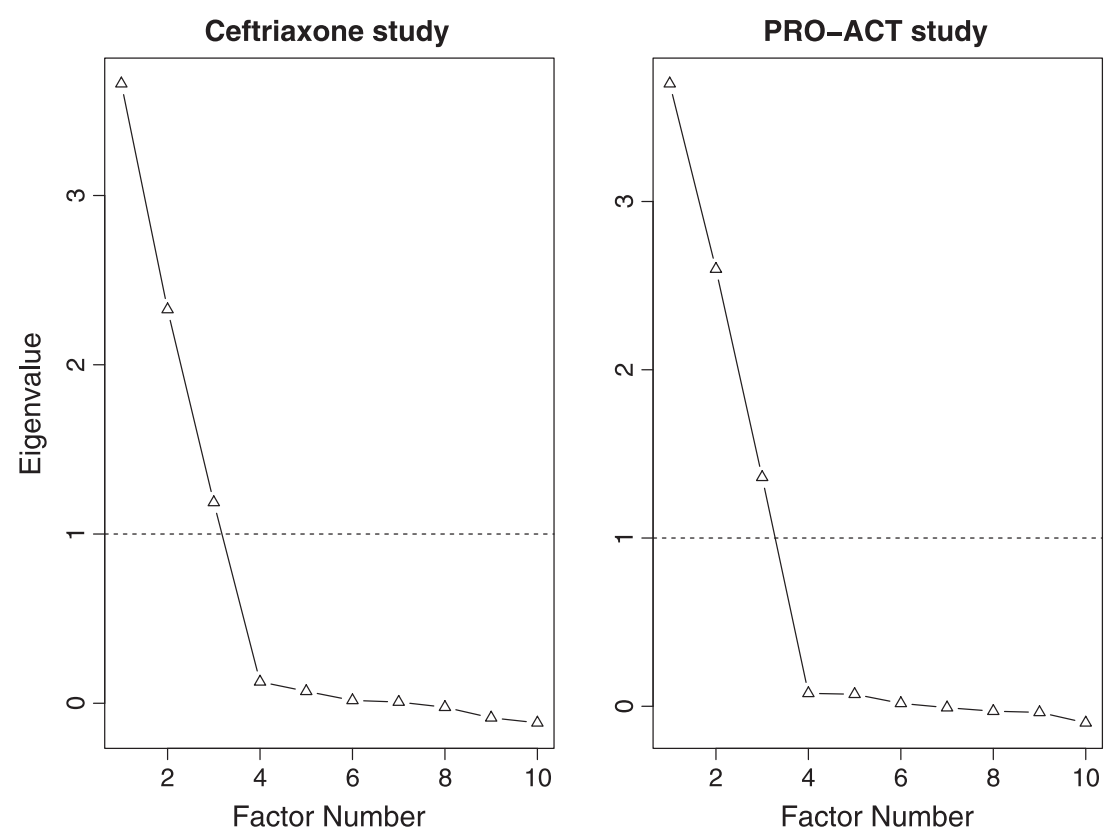

Figure 1. Eigenvalues for exploratory factor analysis. Dotted horizontal line is for eigenvalues equal to 1.

The ALSFRS is the primary outcome in the PRO-ACT challenge data, while the ceftriaxone study uses the revised ALSFRS, which includes two additional questions. To obtain comparable results, the two additional questions are excluded, and the 10-item scores in the original ALSFRS are outcomes of interest in both studies. In the ceftriaxone study, ALSFRS was measured at 21 visits (screening, weeks 4 , weeks 16 , and every 8 weeks starting from weeks 16 to weeks 152 , plus a final study visit performed when subject completed the study). One subject is excluded because of missing ALSFRS at baseline and the final dataset has 512 patients (173 in placebo and 339 in ceftriaxone).

We conduct an exploratory factor analysis and compute the DETECT index [28] based on the baseline ALSFRS data to examine the dimensionality among the 10 items. Figure 1 displays the factor analysis results from both studies. It suggests three factors because the eigenvalues for the first three components are greater than 1 (dotted horizontal lines in Figure 1) [29] and the eigenvalues for the other components are very close to 0 . The factor loading matrix after promax rotation for three factors are displayed in Table S2. Results indicate that (i) items corresponding to the bulbar function (speech, salivation, and swallowing) mainly load on factor 1; (ii) items corresponding to the fine motor function, or mostly upper limb function (handwriting, cutting, and dressing), load on factor 2; and (iii) items corresponding to gross motor function, or mostly lower limb function (walking and climbing), load on factor 3 . Besides the clear-cut factor loadings, Item 7 (turning) overlaps on both the fine motor and gross motor factors (factors 2 and 3) in two studies. Item 10 (breathing) does not highly load on any factor (loadings $<0.4$ ) in the ceftriaxone study but has largest loading of 0.359 on the bulbar factor (factor 1), while it loads on the bulbar factor (with loading of 0.482) in the PRO-ACT study. The results from the DETECT index (Figure S1) also suggests three factors, and the items clustering is similar to factor analysis results. Therefore, the unidimensional assumption may be unreasonable in analyzing the ALSFRS item scores. To this end, we will develop a multidimensional latent trait model to account for the disease impairment in multiple domains.

\section{Model and estimation}

\subsection{Multidimensional latent trait linear mixed model}

Let $y_{i k}(t)$ be the observed outcome $k$ from subject $i$ at time $t$, where $i=1, \ldots, N, k=1, \ldots, K$, and $t=t_{i 1}, \ldots, t_{i J_{i}}$. All outcomes are coded so that larger values are better clinical conditions. To start building the MLTLMM modeling framework, we assume that there are $P$ (with $P<K$ ) LVs representing the underlying disease severity scores and denote them as $\boldsymbol{\theta}_{i}(t)=\left(\theta_{i}^{(1)}(t), \ldots, \theta_{i}^{(p)}(t), \ldots, \theta_{i}^{(P)}(t)\right)^{\prime}$ for subject $i$ at time $t$, where the superscript $(p)(p=1, \ldots, P)$ denotes the $p$ th LV. From a clinical perspective, each LV 
can denote the severity of an ALS impaired domain (e.g., bulbar, fine motor, and gross motor) with lower value indicating worse clinical conditions. We introduce the first level MLTLMM model for continuous outcomes.

$$
y_{i k}(t)=a_{k}+\boldsymbol{b}_{k}^{\prime} \boldsymbol{\theta}_{i}(t)+\varepsilon_{i k}(t),
$$

where $a_{k}$ and $\boldsymbol{b}_{k}=\left(b_{k}^{(1)}, \ldots, b_{k}^{(P)}\right)^{\prime}$ are the outcome-specific parameters and the random errors $\varepsilon_{i k}(t) \sim$ $N\left(0, \sigma_{\varepsilon_{k}}^{2}\right)$ are independent and identically distributed. Note that $a_{k}=E\left[y_{i k}(t) \mid \boldsymbol{\theta}_{i}(t)=\mathbf{0}\right]$ is the mean of the $k$ th outcome if the disease severity scores are 0 and $b_{k}^{(p)}=\left[y_{i k}\left(t^{\prime}\right)-y_{i k}(t)\right] / \theta_{i}^{(p)}(t)$ is the expected increase in the $k$ th outcome for one unit increase in the $p$ th disease severity score while holding other disease severity scores unchanged. The parameter $b_{k}^{(p)}$ also plays the role of bringing up the $p$ th disease severity score to the scale of the $k$ th outcome. When vector $\boldsymbol{b}_{k}$ has different entries, the severity of different ALS impaired domains (latent scores) in $\theta_{i}(t)$ have variable manifestations in the $k$ th outcome. We model outcomes that are binary (e.g., the presence of adverse events) and ordinal (e.g., each item of ALSFRS) using two-parameter submodel [30] and a graded response submodel [31], respectively.

$$
\begin{gathered}
\operatorname{logit}\left\{p\left(y_{i k}(t)=1 \mid \boldsymbol{\theta}_{i}(t)\right)\right\}=a_{k}+\boldsymbol{b}_{k}^{\prime} \boldsymbol{\theta}_{i}(t) \\
\operatorname{logit}\left\{p\left(y_{i k}(t) \leq l \mid \boldsymbol{\theta}_{i}(t)\right)\right\}=a_{k l}-\boldsymbol{b}_{k}^{\prime} \boldsymbol{\theta}_{i}(t),
\end{gathered}
$$

where $l=1,2, \ldots, n_{k}-1$ is the $l$ th level of the $k$ th random variable, which is ordinal with $n_{k}$ levels. Note that the negative sign for $\boldsymbol{b}_{k}$ in the ordinal outcome model is to ensure that worse disease severity (lower $\left.\boldsymbol{\theta}_{i}(t)\right)$ is associated with a more severe outcome (smaller $\left.y_{i k}(t)\right)$. The probability of being in a particular category is $p\left(y_{i k}(t)=l\right)=p\left(y_{i k}(t) \leq l \mid \boldsymbol{\theta}_{i}(t)\right)-p\left(y_{i k}(t) \leq l-1 \mid \boldsymbol{\theta}_{i}(t)\right)$. Interpretation of parameters is similar with continuous outcomes, except that modeling is on the log-odds, not the native scale, of the data. A major feature of these models is that they all incorporate $\boldsymbol{\theta}_{i}(t)$ and explicitly combine information from all outcomes. This is one of the simplest ways to conceptualize the disease severity scores that allows to define the overall treatment effects.

To model the dependence of severity scores $\theta_{i}(t)$ on covariates, we propose the second level multivariate linear mixed model

$$
\theta_{i}^{(p)}(t)=\boldsymbol{X}_{i}^{(p)}(t) \boldsymbol{\beta}^{(p)}+\boldsymbol{Z}_{i}^{(p)}(t) \boldsymbol{u}_{i}^{(p)}+e_{i}^{(p)}(t)
$$

where $\boldsymbol{X}_{i}^{(p)}(t)$ and $\boldsymbol{Z}_{i}^{(p)}(t)$ are the covariates corresponding to fixed and random effects, respectively, for each LV $\theta_{i}^{(p)}(t)$. They can include covariates of interest such as treatment and time. The vector $\boldsymbol{u}_{i}=$ $\left(\boldsymbol{u}_{i}^{(1)^{\prime}}, \ldots, \boldsymbol{u}_{i}^{(P)^{\prime}}\right)^{\prime}$ contains all the random effects for subject $i$, which are assumed to be normally distributed as $N_{P}(0, \boldsymbol{\Sigma})$, where $\boldsymbol{\Sigma}$ is the covariance matrix. The correlation among the LVs are accounted for by the correlation among the elements in $\boldsymbol{u}_{i}$. The residuals $e_{i}^{(p)}(t)$ are assumed to be independent from $\boldsymbol{u}_{i}$ and $e_{i}^{(p)}(t) \sim N\left(0, \sigma_{e}^{(p)^{2}}\right)$.

Models (1) to (4) consist of the MLTLMM model, which provides the framework for defining the overall effects of treatment and other covariates. Indeed, if $\theta_{i}^{(p)}(t)=\beta_{0}^{(p)}+\beta_{1}^{(p)} x_{i}+\beta_{2}^{(p)} t+u_{i}^{(p)}+e_{i}^{(p)}(t)$, where $x_{i}$ is treatment indicator, then $\beta_{1}^{(p)}$ is the treatment effect on the $p$ th underlying disease impairment domain. In this context, the null hypothesis of no overall treatment effect is $H_{0}: \beta_{1}^{(1)}=\cdots=\beta_{1}^{(p)}=$ $\cdots=\beta_{1}^{(P)}=0$. This framework allows not only different treatment effects on multidimensional disease impairment domains to facilitate variable disease progression and prognosis but also the overall effects of the treatment and other covariates to be interpreted on the scale of the observed outcomes. Indeed, on the $k$ th continuous outcome, the effects of the treatment and one unit increase in time are expected to be $\sum_{p=1}^{P} b_{k}^{(p)} \beta_{1}^{(p)}$ and $\sum_{p=1}^{P} b_{k}^{(p)} \beta_{2}^{(p)}$, respectively. Because the number of outcomes $(K)$ has been reduced to a smaller number of latent disease severity scores $(P$, with $P<K)$, models are quite parsimonious in terms of number of random effects, which improves computational feasibility and model interpretability. When there is one LV $(P=1)$, our MLTLMM model reduces to the univariate MLIRT model as a special case.

For notational convenience, we let $\boldsymbol{a}=\left(\boldsymbol{a}_{1}^{\prime}, \ldots, \boldsymbol{a}_{k}^{\prime}, \ldots, \boldsymbol{a}_{K}^{\prime}\right)^{\prime}$, with $\boldsymbol{a}_{k}$ being numeric for binary and continuous outcomes and $\boldsymbol{a}_{k}=\left(a_{k 1}, \ldots, a_{k n_{k}-1}\right)^{\prime}$ for the $k$ th ordinal outcome with $n_{k}$ categories. We let $\boldsymbol{b}=\left(\boldsymbol{b}_{1}, \ldots, \boldsymbol{b}_{K}\right)^{\prime}$, which is the $K$ by $P$ latent factor loading matrix. Then, the disease severity scores $\theta_{i}(t)$ are also known as factor scores (in factor analysis terminology). Because the MLTLMM model is over-parameterized, additional constraints are required to make it identifiable. First, the indeterminacy between the LV loadings $\boldsymbol{b}_{k}$ and the scales of the LVs $\boldsymbol{\theta}_{i}(t)$ can be fixed by either setting one element in each column of $\boldsymbol{b}$ to be 1 , or letting $\sigma_{e}^{(p)}=1$ for $p=1, \ldots, P$ with at least one of the loadings constrained to be positive for each factor [32]. Second, setting the upper diagonal part of $\boldsymbol{b}$ to be 0 (i.e., $b_{k}^{(p)}=0$ for 
all $p>k$ ) fixes the rotation of the latent factor loading matrix and ensures that $\boldsymbol{b}$ and measurement error parameters are identified. For example, when $K=10$ and $P=3$, we can set the identifiability constraints as $b_{1}^{(1)}=b_{2}^{(2)}=b_{3}^{(3)}=1$, and $b_{1}^{(2)}=b_{1}^{(3)}=b_{2}^{(3)}=0$. Finally, to identify parameters $\boldsymbol{a}$ and intercepts in regression coefficients $\boldsymbol{\beta}$, we set $a_{p 1}=0$ for $p=1, \ldots, P$ ordinal outcomes and the order constraint $a_{k 1}<\cdots<a_{k l}<\cdots<a_{k n_{k-1}}$ must be satisfied.

Let the parameter vector $\boldsymbol{\Theta}=\left\{\boldsymbol{a}, \boldsymbol{b}, \boldsymbol{\beta}, \boldsymbol{\Sigma}, \sigma_{\epsilon_{k}}, \sigma_{e}^{(p)}\right\}$. Conditional on the random effects $\boldsymbol{u}_{i}$, all measurements of each subject are assumed to be independent. We have the full likelihood of subject $i$ as follows:

$$
L\left(\boldsymbol{\Theta}, \boldsymbol{u}_{i} ; \boldsymbol{y}_{i}\right)=\left[\prod_{j=1}^{J_{i}} \prod_{k=1}^{K} p\left(y_{i k}\left(t_{i j}\right) \mid \boldsymbol{u}_{i}\right)\right] p\left(\boldsymbol{u}_{i} \mid \mathbf{\Sigma}\right),
$$

where $p\left(\boldsymbol{u}_{i} \mid \mathbf{\Sigma}\right)$ is the density function of random effects vector $\boldsymbol{u}_{i}$. We refer to the univariate MLIRT model as U1LV model and the multivariate MLTLMM model with P LVs as MPLV, for example, M3LV for the MLTLMM model with three LVs.

\subsection{Bayesian inference}

To make inference on the parameter vector $\boldsymbol{\Theta}$, we use Bayesian methods based on Markov chain Monte Carlo (MCMC) posterior simulations. We use vague priors on all elements in $\boldsymbol{\Theta}$, except the aforementioned constrained parameters, that is, $a_{p 1}=0$ and $b_{p}^{(p)}=1$ for $p=1, \ldots, P$, and $b_{k}^{(p)}=0$ for all $p>k$. Specifically, the prior distributions of parameters $a_{k}$ of the continuous outcomes is $a_{k} \sim N(0,100)$. To obtain the prior distributions for the threshold parameters of ordinal outcome $k$, we let $a_{k 1} \sim N(0,100)$, and $a_{k l}=a_{k, l-1}+\Delta_{l}$ for $l=2, \ldots, n_{k}-1$, with $\Delta_{l} \sim N(0,100) I(0$, ), that is, normal distribution left truncated at 0 . Prior distributions for all elements in $\boldsymbol{b}$ (except for aforementioned constrained parameters) and $\boldsymbol{\beta}$ are $N(0,100)$. We use the prior distribution Uniform $[-1,1]$ for all the correlation coefficients $\rho$ in the covariance matrix $\boldsymbol{\Sigma}$, and inverse-gamma $(0.01,0.01)$ for all variance and covariance parameters. We have investigated other selections of vague prior distributions with various hyper-parameters and obtained very similar results.

The posterior samples are obtained from the full conditional of each unknown parameter using Hamiltonian Monte Carlo [33] and No-U-Turn Sampler [34]. Both Hamiltonian Monte Carlo and No-U-Turn Sampler samplers are implemented in Stan, which is a probabilistic programming language implementing statistical inference. The model fitting is performed in Stan (version 2.14.0) [35] by specifying the full likelihood function and the prior distributions of all unknown parameters. For large datasets, Stan may be more efficient than BUGS language [36] in achieving faster convergence and requiring smaller number of samples [34]. Note that Stan requires variable types to be declared prior to modeling. The declaration of matrix $\boldsymbol{\Sigma}$ as a covariance matrix ensures it to be positive-definite by rejecting the samples that cannot produce positive-definite matrix $\boldsymbol{\Sigma}$. To monitor Markov chain convergence, we use the history plots and view the absence of apparent trends in the plot as evidence of convergence. In addition, we use the Gelman-Rubin diagnostic to ensure the scale reduction $\hat{R}$ of all parameters are smaller than 1.1 as well as a suite of convergence diagnosis criteria to ensure convergence [37]. To facilitate easy reading and implementation of the proposed model, a Stan code has been posted in the Supporting Information.

There are a wide variety of model selection criteria in Bayesian inference. Because of the mixture framework in our model, we use the $\mathrm{DIC}_{3}$ measurement [38]. The $\mathrm{DIC}_{3}$ is defined as $\mathrm{DIC}_{3}=$ $\overline{D(\boldsymbol{\Theta})}+\tau_{D}$, where $\overline{D(\boldsymbol{\Theta})}=-2 \mathrm{E}_{\boldsymbol{\Theta} \mid \boldsymbol{D}}\left\{\log \left[\prod_{i=1}^{N} f\left(\boldsymbol{y}_{i} \mid \boldsymbol{\Theta}\right)\right]\right\}$ is the posterior mean deviance, $\tau_{D}=\overline{D(\boldsymbol{\Theta})}+$ $2 \log \left\{\mathrm{E}_{\boldsymbol{\Theta} \mid \boldsymbol{D}}\left[\prod_{i=1}^{N} f\left(\boldsymbol{y}_{i} \mid \boldsymbol{\Theta}\right)\right]\right\}$ is a measure of the effective number of parameters in the model, and $\mathrm{E}_{\boldsymbol{\Theta} \mid \boldsymbol{D}}(\cdot)$ is the expectation with respect to the joint posterior distribution $\pi(\boldsymbol{\Theta} \mid \boldsymbol{D}), \boldsymbol{D}$ denoting the observed data. Thus, we have $\mathrm{DIC}_{3}=-4 \mathrm{E}_{\boldsymbol{\Theta} \mid \boldsymbol{D}}\left\{\log \left[\prod_{i=1}^{N} f\left(\boldsymbol{y}_{i} \mid \boldsymbol{\Theta}\right)\right]\right\}+2 \log \left\{\mathrm{E}_{\boldsymbol{\Theta} \mid \boldsymbol{D}}\left[\prod_{i=1}^{N} f\left(\boldsymbol{y}_{i} \mid \boldsymbol{\Theta}\right)\right]\right\}$. Applying Monte Carlo approximation,

$$
\widehat{\mathrm{DIC}}_{3}=-\frac{4}{M} \sum_{m=1}^{M} \sum_{i=1}^{N} \log \left\{f\left(\boldsymbol{y}_{i} \mid \boldsymbol{\Theta}^{(m)}\right)\right\}+2 \log \left\{\frac{1}{M} \sum_{m=1}^{M} \prod_{i=1}^{N} f\left(\boldsymbol{y}_{i} \mid \boldsymbol{\Theta}^{(m)}\right)\right\},
$$

where $\boldsymbol{\Theta}^{(m)}$ is the $m$ th $(m=1, \ldots, M)$ post burn-in posterior samples of parameter vector $\boldsymbol{\Theta}$. A smaller value of $\mathrm{DIC}_{3}$ indicates a better-fitting model. 


\section{Simulation studies}

In this section, we conduct extensive simulation studies with three settings to investigate the identifiability of the proposed MLTLMM model and also compare its performance with the U1LV model. In all three settings, we generate 200 datasets with sample size $N=600$ subjects and six visits (baseline and five follow-up visits, $J_{i}=6$ ) for each subject. The data structure is similar to the motivating studies with 10 ordinal outcomes $(K=10)$, and each has five categories. Data are simulated from the M2LV model: $\theta_{i}^{(p)}\left(t_{i j}\right)=\beta_{0}^{(p)}+\beta_{1}^{(p)} x_{i}+\beta_{2}^{(p)} t_{i j}+\beta_{3}^{(p)} x_{i} t_{i j}+u_{i}^{(p)}+e_{i}^{(p)}\left(t_{i j}\right)$, where $p=1,2$, the covariate $x_{i}$ takes value 0 or 1 each with probability $1 / 2$ to mimic the treatment assignment. The time vector $\boldsymbol{t}_{i}=\left(t_{i 1}, \ldots, t_{i 6}\right)^{\prime}=$ $(0,1,2,3,4,5)^{\prime}$. We set the regression coefficients to be $\boldsymbol{\beta}^{(1)}=\left(\beta_{0}^{(1)}, \beta_{1}^{(1)}, \beta_{2}^{(1)}, \beta_{3}^{(1)}\right)^{\prime}=(0.5,-1,0.5,-0.4)^{\prime}$ and $\boldsymbol{\beta}^{(2)}=\left(\beta_{0}^{(2)}, \beta_{1}^{(2)}, \beta_{2}^{(2)}, \beta_{3}^{(2)}\right)^{\prime}=(1,-0.5,1,-1)^{\prime}$.

In Setting I, the aim is to confirm that the proposed model is identifiable given the constraints and assumptions in Section 3.1, that is, $a_{11}=a_{21}=0, b_{1}^{(1)}=b_{2}^{(2)}=1$, and $b_{1}^{(2)}=0$, where the true parameters of other elements in vectors $\boldsymbol{a}$ and $\boldsymbol{b}$ are in Tables S3 and S4. Assume that the random effects vector $\boldsymbol{u}_{i}=\left(u_{i}^{(1)}, u_{i}^{(2)}\right)^{\prime} \sim N_{2}(0, \boldsymbol{\Sigma})$, where $\boldsymbol{\Sigma}=\left\{\left(\sigma_{1}^{2}, \rho \sigma_{1} \sigma_{2}\right),\left(\rho \sigma_{1} \sigma_{2}, \sigma_{2}^{2}\right)\right\}$ with $\rho=0.4, \sigma_{1}=1.2$ and $\sigma_{2}=0.8$. The random errors $e_{i}^{(p)}\left(t_{i j}\right) \sim N\left(0, \sigma_{e}^{(p)^{2}}\right)$ with $\sigma_{e}^{(1)}=\sigma_{e}^{(2)}=0.5$. Table I displays bias (the average of the posterior means minus the true values), standard error (SE; the square root of the average of the posterior variance), standard deviation (SD; the $\mathrm{SD}$ of the posterior means), and coverage probabilities $(\mathrm{CP})$ of 95\% equal-tail credible intervals of parameter estimates on $\boldsymbol{\beta}$ and $\boldsymbol{\Sigma}$. The estimates of other parameters are presented in Tables S3 and S4. The results suggest that based on the constraints set on $\boldsymbol{a}$ and $\boldsymbol{b}$, the M2LV model is identifiable, and it provides parameter estimates with negligible biases, SE being close to $\mathrm{SD}$, and the $\mathrm{CP}$ being close to the nominal level 0.95 .

In Setting II, we investigate the performance of the proposed model when identifiability constraints are misspecified. Specifically, we set constraints that $a_{11}=a_{21}=0, b_{1}^{(1)}=b_{2}^{(2)}=1$, and $b_{1}^{(2)}=0$, while the true parameters are $a_{11}=1, a_{21}=0.5, b_{1}^{(1)}=2, b_{1}^{(2)}=0.2$, and $b_{2}^{(2)}=1.8$. This simulation setting is to investigate our proposed model's performance when the true values of those constrained parameters are unknown and are very likely to be unequal to the set constraints. Because the constrained parameters are set to values different from the true parameters, the original parameters has been shifted and rotated. However, it is essential that the true underlying regression relationship between the covariates (e.g., $x$ and $t$ ) and the outcomes (e.g., $\boldsymbol{y}$ ) remain unchanged regardless of shifting and rotation. For example, $\operatorname{logit}\left\{p\left(y_{i 1}(t) \leq l\right)\right\}=a_{1 l}-\boldsymbol{b}_{1}^{\prime} \boldsymbol{\theta}_{i}(t)=a_{1 l}-\sum_{p=1}^{2}\left[b_{1}^{(p)}\left\{\beta_{0}^{(p)}+\beta_{1}^{(p)} x_{i}+\beta_{2}^{(p)} t_{i j}+\beta_{3}^{(p)} x_{i} t_{i j}+u_{i}^{(p)}+e_{i}^{(p)}\left(t_{i j}\right)\right\}\right]$ indicates that the magnitude of underlying regression coefficients between $x_{i}$ and $y_{i 1}$ is $b_{1}^{(1)} \beta_{1}^{(1)}+b_{1}^{(2)} \beta_{1}^{(2)}$. We denote the magnitude of underlying regression coefficients between $x$ and the $k$ th outcome $y_{k}$ as $v_{k}=b_{k}^{(1)} \beta_{1}^{(1)}+b_{k}^{(2)} \beta_{1}^{(2)}$, the underlying association between $t$ and $y_{k}$ as $\gamma_{k}=b_{k}^{(1)} \beta_{2}^{(1)}+b_{k}^{(2)} \beta_{2}^{(2)}$, and the

\begin{tabular}{|c|c|c|c|c|}
\hline & BIAS & SE & SD & $\mathrm{CP}$ \\
\hline$\beta_{0}^{(1)}=0.5$ & 0.009 & 0.088 & 0.098 & 0.915 \\
\hline$\beta_{1}^{(1)}=-1$ & -0.008 & 0.116 & 0.128 & 0.925 \\
\hline$\beta_{2}^{(1)}=0.5$ & -0.001 & 0.020 & 0.021 & 0.925 \\
\hline$\beta_{3}^{(1)}=-0.4$ & 0.001 & 0.024 & 0.026 & 0.925 \\
\hline$\beta_{0}^{(2)}=1$ & 0.001 & 0.082 & 0.081 & 0.950 \\
\hline$\beta_{1}^{(2)}=-0.5$ & 0.004 & 0.108 & 0.110 & 0.950 \\
\hline$\beta_{2}^{(2)}=1$ & -0.005 & 0.046 & 0.047 & 0.940 \\
\hline$\beta_{3}^{(2)}=-1$ & 0.003 & 0.044 & 0.045 & 0.955 \\
\hline$\sigma_{1}=1.2$ & -0.005 & 0.054 & 0.056 & 0.935 \\
\hline$\sigma_{2}=0.8$ & 0.000 & 0.047 & 0.050 & 0.945 \\
\hline$\rho=0.4$ & -0.021 & 0.090 & 0.089 & 0.955 \\
\hline$\sigma_{\rho}^{(1)}=0.5$ & 0.001 & 0.026 & 0.026 & 0.960 \\
\hline$\sigma_{\rho}^{(2)}=0.5$ & 0.003 & 0.027 & 0.031 & 0.925 \\
\hline
\end{tabular}

$\mathrm{SD}$, standard deviation; SE, standard error; $\mathrm{CP}$, coverage probability. 
underlying association between interaction $x \times t$ and $y_{k}$ as $\eta_{k}=b_{k}^{(1)} \beta_{3}^{(1)}+b_{k}^{(2)} \beta_{3}^{(2)}$. Table II (left panel) displays the simulation results for the true underlying coefficients $v_{k}, \gamma_{k}$, and $\eta_{k}$. The parameter estimates from the M2LV model have small biases, SE close to SD, and CP close to 0.95, which suggests that when identifiability assumptions are misspecified, the proposed model still maintains the correct association between covariates and outcomes. In comparison, the U1LV model (right panel) provides considerable biases (e.g., -0.990 for $v_{1}$ ) and poor CP (e.g., 0 for $v_{1}$ ), indicating that unidimensional latent trait model is not sufficient to summarize the variation in the multiple outcomes.

In Setting III, we add one continuous outcome to Setting II. We apply the same misspecified identifiability constraints as in Setting II and summarize the parameter estimates for underlying regression coefficients $v_{k}, \gamma_{k}$, and $\eta_{k}(k=11$ for the additional continuous outcome) in Table S5. The M2LV model provides good fits for mixed types of outcomes with small biases and CP close to $95 \%$.

In summary, we conclude that the proposed MLTLMM model is identifiable regardless whether the identifiability constraints are set at the true values. In the presence of multiple LVs, the MLTLMM model provides satisfactory results, while the U1LV model results in considerable bias and poor CP. The MLTLMM model also provides good performance when both ordinal and continuous outcomes exist.

\begin{tabular}{|c|c|c|c|c|c|c|c|c|}
\hline & \multicolumn{4}{|c|}{ M2LV } & \multicolumn{4}{|c|}{ U1LV } \\
\hline & BIAS & SE & SD & $\mathrm{CP}$ & BIAS & SE & $\mathrm{SD}$ & $\mathrm{CP}$ \\
\hline \multicolumn{9}{|c|}{ Coefficients for covariate $x$} \\
\hline$v_{1}=2.1$ & 0.003 & 0.238 & 0.230 & 0.935 & -0.990 & 0.115 & 0.122 & 0.000 \\
\hline$v_{2}=1.1$ & 0.015 & 0.159 & 0.151 & 0.960 & 0.279 & 0.141 & 0.128 & 0.480 \\
\hline$v_{3}=1.7$ & 0.015 & 0.186 & 0.183 & 0.960 & -0.525 & 0.121 & 0.126 & 0.020 \\
\hline$v_{4}=2.05$ & 0.020 & 0.238 & 0.239 & 0.950 & -1.032 & 0.106 & 0.114 & 0.000 \\
\hline$v_{5}=0.9$ & 0.013 & 0.139 & 0.138 & 0.950 & 0.325 & 0.125 & 0.118 & 0.225 \\
\hline$v_{6}=1.3$ & 0.009 & 0.145 & 0.137 & 0.955 & -0.428 & 0.090 & 0.092 & 0.010 \\
\hline$v_{7}=1.75$ & 0.014 & 0.194 & 0.187 & 0.960 & -0.636 & 0.114 & 0.118 & 0.005 \\
\hline$v_{8}=0.8$ & 0.013 & 0.111 & 0.107 & 0.965 & 0.293 & 0.112 & 0.105 & 0.210 \\
\hline$v_{9}=0.8$ & 0.008 & 0.101 & 0.102 & 0.965 & 0.242 & 0.106 & 0.104 & 0.365 \\
\hline$v_{10}=1.15$ & 0.019 & 0.146 & 0.141 & 0.970 & 0.299 & 0.148 & 0.136 & 0.455 \\
\hline \multicolumn{9}{|c|}{ Coefficients for covariate $t$} \\
\hline$\gamma_{1}=-1.2$ & 0.003 & 0.038 & 0.034 & 0.975 & 0.141 & 0.028 & 0.029 & 0.000 \\
\hline$\gamma_{2}=-1.9$ & -0.005 & 0.058 & 0.061 & 0.945 & 0.582 & 0.038 & 0.050 & 0.000 \\
\hline$\gamma_{3}=-1.15$ & -0.002 & 0.033 & 0.035 & 0.945 & 0.030 & 0.028 & 0.030 & 0.825 \\
\hline$\gamma_{4}=-1.1$ & -0.004 & 0.037 & 0.038 & 0.945 & 0.130 & 0.025 & 0.028 & 0.000 \\
\hline$\gamma_{5}=-1.65$ & -0.008 & 0.048 & 0.048 & 0.955 & 0.480 & 0.032 & 0.040 & 0.000 \\
\hline$\gamma_{6}=-0.8$ & -0.002 & 0.025 & 0.026 & 0.935 & -0.032 & 0.022 & 0.023 & 0.680 \\
\hline$\gamma_{7}=-1.1$ & -0.002 & 0.033 & 0.030 & 0.950 & 0.038 & 0.027 & 0.028 & 0.675 \\
\hline$\gamma_{8}=-1.3$ & -0.005 & 0.035 & 0.035 & 0.955 & 0.256 & 0.028 & 0.030 & 0.000 \\
\hline$\gamma_{9}=-1.15$ & -0.002 & 0.030 & 0.028 & 0.970 & 0.156 & 0.026 & 0.029 & 0.000 \\
\hline$\gamma_{10}=-1.7$ & -0.008 & 0.047 & 0.041 & 0.980 & 0.316 & 0.038 & 0.043 & 0.000 \\
\hline \multicolumn{9}{|c|}{ Coefficients for interaction $x \times t$} \\
\hline$\eta_{1}=1$ & -0.004 & 0.043 & 0.040 & 0.950 & -0.004 & 0.028 & 0.029 & 0.940 \\
\hline$\eta_{2}=1.88$ & 0.008 & 0.062 & 0.064 & 0.925 & -0.640 & 0.039 & 0.050 & 0.000 \\
\hline$\eta_{3}=1$ & 0.000 & 0.036 & 0.038 & 0.945 & 0.054 & 0.029 & 0.032 & 0.520 \\
\hline$\eta_{4}=0.9$ & 0.002 & 0.042 & 0.041 & 0.960 & 0.013 & 0.026 & 0.027 & 0.920 \\
\hline$\eta_{5}=1.64$ & 0.010 & 0.052 & 0.052 & 0.955 & -0.539 & 0.034 & 0.041 & 0.000 \\
\hline$\eta_{6}=0.68$ & 0.001 & 0.028 & 0.029 & 0.935 & 0.103 & 0.022 & 0.023 & 0.000 \\
\hline$\eta_{7}=0.94$ & 0.000 & 0.037 & 0.034 & 0.970 & 0.059 & 0.028 & 0.028 & 0.440 \\
\hline$\eta_{8}=1.28$ & 0.006 & 0.038 & 0.039 & 0.965 & -0.298 & 0.029 & 0.032 & 0.000 \\
\hline$\eta_{9}=1.12$ & 0.003 & 0.032 & 0.032 & 0.945 & -0.184 & 0.027 & 0.030 & 0.000 \\
\hline$\eta_{10}=1.66$ & 0.009 & 0.050 & 0.044 & 0.960 & -0.358 & 0.039 & 0.045 & 0.000 \\
\hline
\end{tabular}

$\mathrm{SD}$, standard deviation; SE, standard error; $\mathrm{CP}$, coverage probability. 


\section{Real data analysis}

In this section, we apply the proposed MLTLMM model and the Bayesian inference framework to the motivating ceftriaxone and PRO-ACT studies with outcomes being the 10 items in ALSFRS. Specifically, we compare the U1LV model with the MLTLMM models with various numbers of LVs. Bayesian model selection criteria such as DIC are used to determine the number of LVs and to select the final model. For all the results in this section, we run two parallel MCMC chains with overdispersed initial values and run each chain for 2000 iterations. The first 1000 iterations are discarded as burn-in, and the inference is based on the remaining 1000 iterations from each chain. Good mixing properties of the MCMC chains for all model parameters are observed in the trace plots. The scale reduction $\hat{R}$ of all parameters are smaller than 1.1 .

\subsection{Ceftriaxone study}

In the ceftriaxone study, we consider the following covariates for all LVs: the treatment assignment variable $\operatorname{trt}_{i}$ ( 1 for ceftriaxone, and 0 for placebo), time $t_{i j}$ in years, and time and treatment interaction. Hence, Model (4) is $\theta_{i}^{(p)}\left(t_{i j}\right)=\beta_{0}^{(p)}+\beta_{1}^{(p)} \operatorname{trt}_{i}+\beta_{2}^{(p)} t_{i j}+\beta_{3}^{(p)}\left(\operatorname{trt}_{i} \times t_{i j}\right)+u_{i}^{(p)}+e_{i}^{(p)}\left(t_{i j}\right)$, where $\boldsymbol{u}_{i}=\left(u_{i}^{(1)}, \ldots, u_{i}^{(P)}\right)^{\prime} \sim N(0, \Sigma)$, with $\Sigma_{r r}=\sigma_{r}^{2}, \Sigma_{r s}=\rho_{r s} \sigma_{r} \sigma_{s}$, and $r, s=1, \ldots, P$. For identifiability and the ease of interpretation of the MLTLMM model with $P$ LVs, we select $P$ items (out of a total of 10 items) and assume that each of the selected item loads on only one LV. For example, to fit the M3LV model, we impose identifiability constraints on one item from each cluster, that is, Item 1 (speech), Item 5 (cutting), and Item 8 (walking), by letting $a_{11}=a_{51}=a_{81}=0$, diagonal elements of factor loading matrix $\left(\boldsymbol{b}_{1}, \boldsymbol{b}_{5}, \boldsymbol{b}_{8}\right)^{\prime}$ equal to 1 and off-diagonal elements equal to 0 .

We compare the U1LV model with various MLTLMM models using Bayesian model selection criteria. Figure 2 (left panel) displays the DIC for the U1LV model and the MLTLMM models with two to five LVs. Using the M5LV model as a reference, we compute the ratios of the DICs from the models with one to four LVs to the DIC from the M5LV model. Extending from unidimensional to multidimensional models, we obtain a significant improvement in model fitting in terms of DIC (e.g., DIC ratio 1.39 vs 1.20 for the U1LV and M2LV models, respectively). The M3LV model also has much smaller DIC than the M2LV model (1.06 vs 1.20). However, the gain of goodness of fit by using more than three LVs is marginal, with DIC ratio 1.06 vs 1.02 comparing the M3LV and M4LV models, and 1.02 vs 1.00 comparing the M4LV and M5LV models. Visually speaking, the last significant drop or break takes place at the M3LV model, which is similar in spirit to Cattell's scree test [39]. Because the MLTLMM models with more LVs have limited improvement in goodness of fit but more complexity and computational challenge, the M3LV model is selected as the final model.

Table III (left panel) presents the factor loading matrix for the selected M3LV model. Item 1 (speech), Item 5 (cutting), and Item 8 (walking) are restricted on $\theta^{(1)}, \theta^{(2)}$, and $\theta^{(3)}$, respectively, as the identifiability constraints. Item 2 (salivation) and Item 3 (swallowing) entirely load on $\theta^{(1)}$ with loadings of 0.496 and 0.623 , respectively, while the magnitude of their loadings on $\theta^{(2)}$ and $\theta^{(3)}$ are negligible $(<0.1)$. Similarly, Item 4 (handwriting) and Item 6 (dressing) mainly load on $\theta^{(2)}$, and Item 9 (climbing) generally loads on $\theta^{(3)}$. Item 7 (turning) cross loads on both $\theta^{(2)}$ and $\theta^{(3)}$ but with slightly larger loading on $\theta^{(2)}(0.355$ vs 0.314 ). Item 10 (breathing) does not highly load on any of the LVs but with largest loading of 0.120 on $\theta^{(1)}$. Hence, the results from the M3LV model are consistent with the exploratory factor analysis, indicating that the three $\operatorname{LVs} \theta^{(1)}, \theta^{(2)}$, and $\theta^{(3)}$ are the underlying disease severity associated with bulbar, fine motor, and gross motor functions, respectively. Moreover, Item 7 overlaps on the fine motor and gross motor functions, while Item 10 slightly loads to the bulbar function.

Table IV (left panel) displays the posterior mean, SD, and 95\% equal-tail credible intervals for the M3LV model. Ceftriaxone is not significant in all three LVs. Specifically, ceftriaxone is associated with 0.643 unit decrease $\left(\hat{\beta}_{1}^{(1)}, 95 \%\right.$ CI: $\left.[-1.694,0.296]\right)$ in the bulbar function, 0.337 unit decrease $\left(\hat{\beta}_{1}^{(2)}, 95 \%\right.$ CI: $[-1.221,0.587])$ in the fine motor function, and 0.455 unit increase $\left(\hat{\beta}_{1}^{(3)}, 95 \%\right.$ CI: $\left.[-0.686,1.526]\right)$ in the gross motor function. The placebo patients have significant ALS progression at the rate of -2.829 unit per year $\left(\hat{\beta}_{2}^{(1)}, 95 \%\right.$ CI: $\left.[-3.102,-2.579]\right)$ in the bulbar function, -4.347 unit per year $\left(\hat{\beta}_{2}^{(2)}, 95 \%\right.$ CI: $[-4.701,-4.022])$ in the fine motor function, and -4.746 unit per year $\left(\hat{\beta}_{2}^{(3)}, 95 \%\right.$ CI: $\left.[-5.203,-4.296]\right)$ in the gross motor function. In comparison, ceftriaxone patients have ALS progression rate of -2.608 unit per year $\left(\hat{\beta}_{2}^{(1)}+\hat{\beta}_{3}^{(1)}, 95 \%\right.$ CI: $\left.[-3.102,-2.133]\right)$ with borderline significant ceftriaxone treatment 

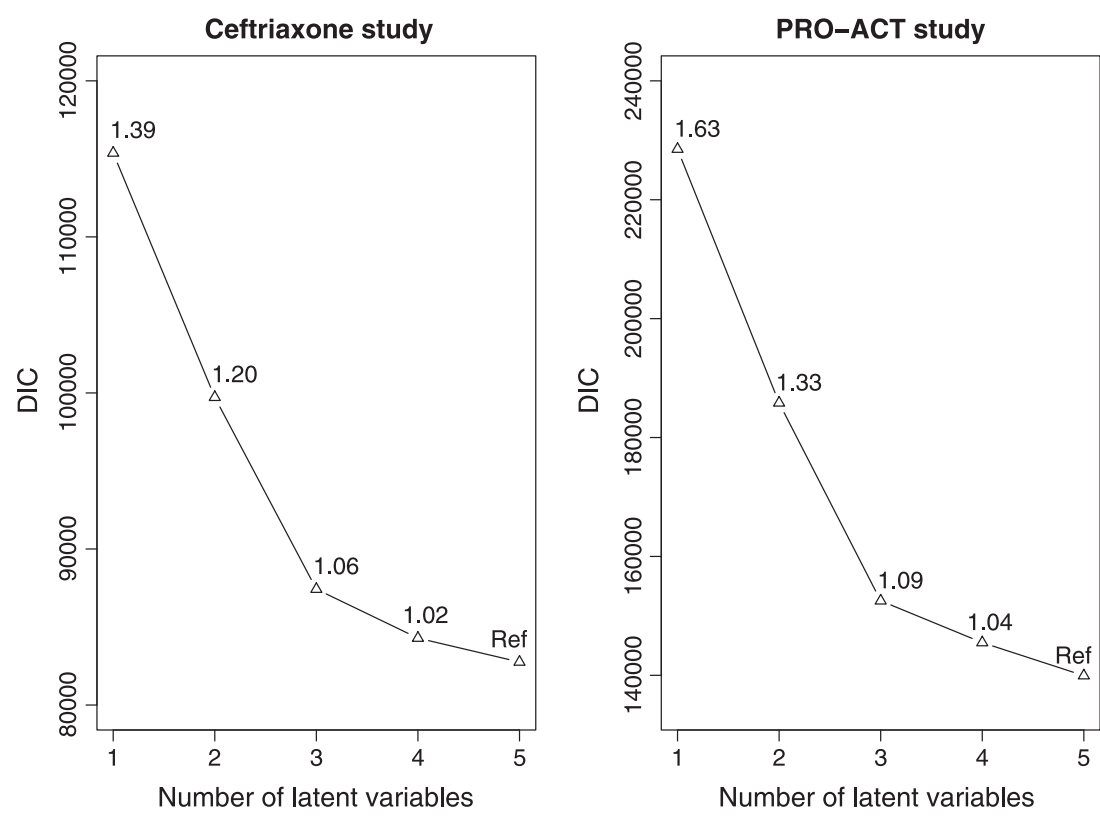

Figure 2. DIC plot for the ceftriaxone study (left panel) and PRO-ACT study (right panel). Numbers on the figure are DIC ratios of models with one to four latent variables to the M5LV reference model.

\begin{tabular}{|c|c|c|c|c|c|c|}
\hline & \multicolumn{3}{|c|}{ Ceftriaxone study } & \multicolumn{3}{|c|}{ PRO-ACT study } \\
\hline & $\theta^{(1)}$ & $\theta^{(2)}$ & $\theta^{(3)}$ & $\theta^{(1)}$ & $\theta^{(2)}$ & $\theta^{(3)}$ \\
\hline 1. Speech & 1 & 0 & 0 & 1 & 0 & 0 \\
\hline 2. Salivation & 0.496 & 0.009 & -0.034 & 0.477 & 0.004 & -0.014 \\
\hline 3. Swallowing & 0.623 & 0.038 & 0.058 & 0.539 & 0.054 & 0.020 \\
\hline 4. Handwriting & -0.021 & 0.623 & 0.008 & -0.013 & 0.716 & -0.026 \\
\hline 5. Cutting food & 0 & 1 & 0 & 0 & 1 & 0 \\
\hline 6. Dressing and hygiene & -0.022 & 0.591 & 0.200 & -0.046 & 0.625 & 0.230 \\
\hline 7. Turning in bed & 0.040 & 0.355 & 0.314 & -0.010 & 0.395 & 0.312 \\
\hline 8. Walking & 0 & 0 & 1 & 0 & 0 & 1 \\
\hline 9. Climbing stairs & 0.031 & 0.089 & 0.759 & 0.019 & 0.103 & 0.931 \\
\hline 10. Breathing & 0.120 & 0.044 & 0.092 & 0.149 & 0.044 & 0.071 \\
\hline
\end{tabular}

PRO-ACT, Pooled Resource Open-Access ALS Clinical Trials.

Boldface numbers indicate the factor loading structure.

effect of slowing down the ALS progression rate by 0.221 per year $\left(\hat{\beta}_{3}^{(1)}, 95 \%\right.$ CI: $\left.[0.000,0.446]\right)$ in the bulbar function. Similar interpretations can be made for the other two LVs.

The estimates of the correlation coefficients $\rho_{12}, \rho_{13}$, and $\rho_{23}$ are 0.259 (95\% CI: [0.179, 0.339]), 0.074 (95\% CI: $[-0.018,0.161])$, and 0.446 (95\% CI: $[0.373,0.520])$, respectively. The significant positive correlations of $\theta^{(1)}$ and $\theta^{(2)}$, and of $\theta^{(2)}$ and $\theta^{(3)}$ indicate that the ALS patients with poor fine motor function (lower $u_{i}^{(2)}$ ) tend to have poor bulbar (lower $u_{i}^{(1)}$ ) and gross motor (lower $u_{i}^{(3)}$ ) functions. However, the bulbar and gross motor functions are less correlated. For completeness, the parameters $\boldsymbol{a}$ and $\boldsymbol{b}$ are presented in Tables S6 and S7, respectively.

\subsection{PRO-ACT study}

We apply all models to the PRO-ACT study. The DIC plot in Figure 2 (right panel) also suggests that M3LV model as the final model because it offers a nice balance between model goodness of fit and complexity. Table III (right panel) displays the factor loading matrix for the M3LV model. Although the parameter estimates in two studies are different, items are classified into the same three domains, that is, bulbar function (including items of speech, salivation, and swallowing), fine motor function (includ- 


\begin{tabular}{|c|c|c|c|c|c|c|c|c|}
\hline & \multicolumn{4}{|c|}{ Ceftriaxone study } & \multicolumn{4}{|c|}{ PRO-ACT study } \\
\hline & Mean & SD & \multicolumn{2}{|c|}{$95 \% \mathrm{CI}$} & Mean & SD & \multicolumn{2}{|c|}{$95 \% \mathrm{CI}$} \\
\hline \multicolumn{9}{|c|}{ Parameters for the bulbar function } \\
\hline $\operatorname{Int}\left(\beta_{0}^{(1)}\right)$ & 8.566 & 0.470 & 7.658 & 9.579 & 13.523 & 0.560 & 12.379 & 14.575 \\
\hline $\operatorname{Trt}\left(\beta_{1}^{(1)}\right)$ & -0.643 & 0.503 & -1.694 & 0.296 & -0.811 & 0.573 & -1.827 & 0.418 \\
\hline Time $\left(\beta_{2}^{(1)}\right)$ & -2.829 & 0.130 & -3.102 & -2.579 & -4.616 & 0.145 & -4.893 & -4.339 \\
\hline Time:Trt $\left(\beta_{3}^{(1)}\right)$ & 0.221 & 0.113 & 0.000 & 0.446 & 1.043 & 0.130 & 0.789 & 1.292 \\
\hline \multicolumn{9}{|c|}{ Parameters for the fine motor function } \\
\hline $\operatorname{Int}\left(\beta_{0}^{(2)}\right)$ & 7.378 & 0.473 & 6.480 & 8.326 & 10.725 & 0.447 & 9.849 & 11.629 \\
\hline $\operatorname{Trt}\left(\beta_{1}^{(2)}\right)$ & -0.337 & 0.477 & -1.221 & 0.587 & -0.352 & 0.467 & -1.267 & 0.529 \\
\hline Time $\left(\beta_{2}^{(2)}\right)$ & -4.347 & 0.173 & -4.701 & -4.022 & -5.234 & 0.152 & -5.530 & -4.940 \\
\hline Time:Trt $\left(\beta_{3}^{(2)}\right)$ & 0.196 & 0.126 & -0.056 & 0.443 & 0.631 & 0.128 & 0.384 & 0.889 \\
\hline \multicolumn{9}{|c|}{ Parameters for the gross motor function } \\
\hline $\operatorname{Int}\left(\beta_{0}^{(3)}\right)$ & 11.382 & 0.625 & 10.103 & 12.538 & 13.419 & 0.498 & 12.485 & 14.394 \\
\hline $\operatorname{Trt}\left(\beta_{1}^{(3)}\right)$ & 0.455 & 0.591 & -0.686 & 1.526 & 0.576 & 0.472 & -0.357 & 1.495 \\
\hline Time $\left(\beta_{2}^{(3)}\right)$ & -4.746 & 0.234 & -5.203 & -4.296 & -5.036 & 0.163 & -5.337 & -4.702 \\
\hline Time:Trt $\left(\beta_{3}^{(3)}\right)$ & 0.034 & 0.158 & -0.263 & 0.359 & 0.133 & 0.139 & -0.136 & 0.399 \\
\hline$\sigma_{1}$ & 5.270 & 0.244 & 4.823 & 5.784 & 7.422 & 0.261 & 6.908 & 7.940 \\
\hline$\sigma_{2}$ & 5.014 & 0.221 & 4.600 & 5.463 & 5.993 & 0.190 & 5.646 & 6.366 \\
\hline$\sigma_{3}$ & 6.013 & 0.308 & 5.406 & 6.635 & 6.511 & 0.216 & 6.092 & 6.933 \\
\hline$\rho_{12}$ & 0.259 & 0.041 & 0.179 & 0.339 & 0.198 & 0.033 & 0.134 & 0.262 \\
\hline$\rho_{13}$ & 0.074 & 0.046 & -0.018 & 0.161 & -0.047 & 0.035 & -0.118 & 0.020 \\
\hline$\rho_{23}$ & 0.446 & 0.037 & 0.373 & 0.520 & 0.284 & 0.031 & 0.223 & 0.345 \\
\hline
\end{tabular}

PRO-ACT, Pooled Resource Open-Access ALS Clinical Trials; SD, standard deviation; CI, confidence interval.

ing items of handwriting, cutting, and dressing), and gross motor function (including items of walking, climbing), while Item 7 (turning) overlaps on the fine motor and gross motor functions and Item 10 (breathing) slightly loads on the bulbar function.

Table IV (right panel) displays the estimates of regression coefficients from the M3LV model. Comparing with the ceftriaxone study, we detect significant effects of treatment and time interaction in the bulbar and fine motor functions, that is, the treatment significantly slows down the ALS progression by 1.043 unit per year $\left(\hat{\beta}_{3}^{(1)}, 95 \%\right.$ CI: $\left.[0.789,1.292]\right)$ in the bulbar function, and 0.631 unit per year $\left(\hat{\beta}_{3}^{(2)}\right.$, 95\% CI: $[0.384,0.889]$ ) in the fine motor function, but insignificant treatment effect in the gross motor function. This new finding signifies the unique advantage of our MLTLMM framework: allow different covariate effects on various ALS impaired domains to facilitate variable progression and prognosis. In comparison, the unidimensional MLIRT model or models based on the ALSFRS total score can only provide the overall covariate effects. One caveat about the PRO-ACT study is that we use the challenge data, which is a subset of the PRO-ACT database consisting of 23 ALS clinical trials with various treatments. Therefore, the significant treatment effects in some ALS domains need to be validated in other ALS studies. For completeness, the parameters $\boldsymbol{a}$ and $\boldsymbol{b}$ are presented in Tables S8 and S9, respectively.

\section{Discussion}

In this article, we propose a MLTLMM for multivariate longitudinal outcomes of mixed types (e.g., categorical and continuous). The proposed model allows for multiple LVs and within-item multidimensionality (i.e., one outcome can be a manifestation of more than one LV). We adopt a Bayesian inference framework based on the MCMC simulation for parameter estimation. The extensive simulation studies suggest that the MLTLMM model is identifiable regardless whether the identifiability constraints are set at the true values. In the presence of multidimensionality, the MLTLMM model provides reasonable estimates, while the widely used unidimensional MLIRT model provides invalid results. We apply 
the MLTLMM model to two motivating ALS studies. The MLTLMM model has identified three LVs representing multiple ALS domains in the bulbar, fine motor, and gross motor functions. The unique advantage of the MLTLMM framework is that it allows different covariate effects on various disease domains to facilitate variable progression and prognosis. The proposed model can be implemented using the publicly available software packages such as BUGS and Stan and can be readily accessible to applied researchers.

Of note, our MLTLMM model is identified by setting the following identification constraints on some outcome-specific parameters: $a_{p 1}=0$ and $b_{p}^{(p)}=1$ for $p=1, \ldots, P$ and $b_{k}^{(p)}=0$ for all $p>k$. One limitation of these constraints is that the relationships between item parameters $\boldsymbol{a}$ and $\boldsymbol{b}$ cannot be specified a priori. Alternatively, the identification constraints can be set on the latent scores by restricting directly their means and covariances. While in the univariate LV model it may be reasonable to set the mean and variance of the LV to some fixed values, setting the means and covariances of multiple LVs to some known values and how to select those values would be challenging and lead to interpretation difficulty.

There are some limitations in the MLTLMM model that we will address in our future study. First, the method for determining the number of LVs involves the ratio of DICs between two candidate models and a graphical representation of the DICs to determine the point at which the last significant drop takes place, similar in spirit to Cattell's scree test [39]. However, the DIC is a rough guideline in choosing the best model, and it cannot provide accurate information about which model fits the data best. A more objective way of determining the number of LVs will be further investigated. Second, as pointed out by a reviewer, an underlying assumption of the proposed model is that the treatment effects on the individual disease symptoms or items can be fully captured by the latent traits. However, there might be residual direct effects of the treatment on specific symptoms. For example, Item 10 (breathing) does not highly load on any of three LVs, and hence, the treatment effects on this item through LVs are not easily interpretable. But this item may reflect a clinically important symptom of ALS, and thus, the direct treatment effects on breathing may be of clinical interest. We would relax this assumption by modeling treatment effects in both the latent traits and as another covariate in the measurement model (i.e., models (1) and (2)) in future study. Third, both the monotone (e.g., dropout) and non-monotone (e.g., missed visit) missing exist in the ALS studies. In this article, we assume they are missing at random. However, monotone missing is likely to be caused by some terminal events (e.g., death), which are often correlated with the health outcomes such as ALSFRS and they create the issue of informative censoring. How to address the informative censoring issue in the proposed MLTLMM model is an interesting direction of future research. Moreover, when the non-monotone missingness due to missed visit is associated with either the unobserved value or the underlying health status (e.g., sicker patients are more likely to miss visits), it is missing not at random [40]. Under the missing not at random assumption, the missing data mechanism needs to be modeled simultaneously with the outcome variable to avoid biased parameter estimates [41].

We assume measurement invariance in our model, that is, the outcome-specific parameters $a_{k}$ and $\boldsymbol{b}_{k}$ are invariant over occasions and over various specified variables such as gender, ethnicity. However, this assumption may be invalid in the presence of item parameter shift [42] (i.e., $a_{k}$ and $\boldsymbol{b}_{k}$ change over occasions) and differential item functioning (i.e., the ALSFRS items may measure different ALS domains for members of separate subgroups). How to test for measurement invariance in our model warrants further investigation. Moreover, we have chosen a multivariate normal distribution for the random effects vector because it is flexible in modeling the covariance structure within and between various LVs, and it has meaningful interpretation on correlation. In generalized linear mixed models, misspecification of random effects distribution has little impact on the parameters that are not associated with the random effects [43]. The impact of random effects misspecification in the proposed modeling framework warrants further investigation. We will also investigate the effect of random effects misspecification and relax the normality assumption by considering Bayesian non-parametric framework based on Dirichlet process mixture [44].

\section{Acknowledgements}

Sheng Luo's research was supported in part by the National Institute of Neurological Disorders and Stroke under award numbers R01NS091307 and 5U01NS043127. Data used in the preparation of this article were obtained from the Pooled Resource Open-Access ALS Clinical Trials (PRO-ACT) database. As such, the following organizations and individuals within the PRO-ACT consortium contributed to the design and implementation of the PRO-ACT database and/or provided data, but did not participate in the analysis of the data or the writing of this report: Neurological Clinical Research Institute, MGH; Northeast ALS Consortium; Novartis; Prize4Life; Regen- 
eron Pharmaceuticals, Inc.; Sanofi; and Teva Pharmaceutical Industries, Ltd. The authors acknowledge the Texas Advanced Computing Center (TACC) at The University of Texas at Austin for providing high-performing computing resources that have contributed to the research results reported within this article. URL:http://www.tacc. utexas.edu.

\section{References}

1. Kiernan MC, Vucic S, Cheah BC, Turner MR, Eisen A, Hardiman O, Burrell JR, Zoing MC. Amyotrophic lateral sclerosis. The Lancet 2011; 377(9769):942-955.

2. Berry JD, Shefner JM, Conwit R, Schoenfeld D, Keroack M, Felsenstein D, Krivickas L, David WS, Vriesendorp F, Pestronk A, Caress JB, Katz J, Simpson E, Rosenfeld J, Pascuzzi R, Glass J, Rezania K, Rothstein JD, Greenblatt DJ, Cudkowicz ME, Northeast ALS Consortium. Design and initial results of a multi-phase randomized trial of ceftriaxone in amyotrophic lateral sclerosis. PLoS One 2013; 8(4):e61177.

3. Pooled Resource Open-Access ALS Clinical Trials Database. Data Sets. 2013 (December 18, 2013). Available from: https://nctu.partners.org/ProACT/Data.

4. Abe K, Itoyama Y, Sobue G, Tsuji S, Aoki M, Doyu M, Hamada C, Kondo K, Yoneoka T, Akimoto M, Yoshino H, Edaravone ALS Study Group. Confirmatory double-blind, parallel-group, placebo-controlled study of efficacy and safety of edaravone (MCI-186) in amyotrophic lateral sclerosis patients. Amyotrophic Lateral Sclerosis and Frontotemporal Degeneration 2014; 15(7-8):610-617.

5. Clarke S, Hickey A, O'boyle C, Hardiman O. Assessing individual quality of life in amyotrophic lateral sclerosis. Quality of Life Research 2001; 10(2):149-158.

6. Coco D, Marchese S, La Bella V, Piccoli T, Coco A. The amyotrophic lateral sclerosis functional rating scale predicts survival time in amyotrophic lateral sclerosis patients on invasive mechanical ventilation. Chest Journal 2007; 132(1): 64-69.

7. Bedlack RS, Vaughan T, Wicks P, Heywood J, Sinani E, Selsov R, Macklin EA, Schoenfeld D, Cudkowicz M, Sherman A. How common are ALS plateaus and reversals?. Neurology 2016; 86(9):808-812.

8. The ACTS Study Group. The amyotrophic lateral sclerosis functional rating scale. Assessment of activities of daily living in patients with amyotrophic lateral sclerosis. Archives of Neurology 1996; 53(2):141-147.

9. Proust-Lima C, Amieva H, Jacqmin-Gadda H. Analysis of multivariate mixed longitudinal data: a flexible latent process approach. British Journal of Mathematical and Statistical Psychology 2013; 66(3):470-487.

10. van den Hout A, Fox JP, Muniz-Terrera G. Longitudinal mixed-effects models for latent cognitive function. Statistical Modelling 2015; 15(4):366-387.

11. Gorter R, Fox JP, Twisk JW. Why item response theory should be used for longitudinal questionnaire data analysis in medical research. BMC Medical Research Methodology 2015; 15(1):55-66.

12. Fox J, Glas C. Bayesian estimation of a multilevel IRT model using Gibbs sampling. Psychometrika 2001; 66(2):271-288.

13. Glas C, Geerlings H, van de Laar M, Taal E. Analysis of longitudinal randomized clinical trials using item response models. Contemporary Clinical Trials 2009; 30(2):158-170.

14. Luo S, Wang J. Bayesian hierarchical model for multiple repeated measures and survival data: an application to Parkinson's disease. Statistics in Medicine 2014; 33(24):4279-4291.

15. Bacci ED, Staniewska D, Coyne KS, Boyer S, White LA, Zach N, Cedarbaum JM, Pooled Resource Open-Access ALS Clinical Trials Consortium. Item response theory analysis of the amyotrophic lateral sclerosis functional rating scalerevised in the pooled resource open-access ALS clinical trials database. Amyotrophic Lateral Sclerosis and Frontotemporal Degeneration 2016; 17(3-4):157-167.

16. Verhagen J, Fox JP. Longitudinal measurement in health-related surveys. A Bayesian joint growth model for multivariate ordinal responses. Statistics in Medicine 2013; 32(17):2988-3005.

17. Schmidt S, Zlatkin-Troitschanskaia O, Fox JP. Pretest-posttest-posttest multilevel IRT modeling of competence growth of students in higher education in Germany. Journal of Educational Measurement 2016; 53(3):332-351.

18. Franchignoni F, Mora G, Giordano A, Volanti P, Chiò A. Evidence of multidimensionality in the ALSFRS-R Scale: a critical appraisal on its measurement properties using Rasch analysis. Journal of Neurology, Neurosurgery \& Psychiatry $2013 ; \mathbf{8 4}(12): 1340-1345$.

19. te Marvelde JM, Glas CA, Van Landeghem G, Van Damme J. Application of multidimensional item response theory models to longitudinal data. Educational and Psychological Measurement 2006; 66(1):5-34.

20. Cho SJ, Athay M, Preacher KJ. Measuring change for a multidimensional test using a generalized explanatory longitudinal item response model. British Journal of Mathematical and Statistical Psychology 2013; 66(2):353-381.

21. Cho SJ, Cohen AS. A multilevel mixture IRT model with an application to DIF. Journal of Educational and Behavioral Statistics 2010; 35(3):336-370.

22. Fox JP, Entink RK, Avetisyan M. Compensatory and non-compensatory multidimensional randomized item response models. British Journal of Mathematical and Statistical Psychology 2014; 67(1):133-152.

23. Klein Entink R, Fox JP, Van Der Linden W. A multivariate multilevel approach to the modeling of accuracy and speed of test takers. Psychometrika 2009; 74(1):21-48.

24. Fox JP, Marianti S. Joint modeling of ability and differential speed using responses and response times. Multivariate Behavioral Research 2016; 51(4):540-553.

25. Muthén B, Curran P. General longitudinal modeling of individual differences in experimental designs: a latent variable framework for analysis and power estimation. Psychological Methods 1997; 2(4):371-402.

26. Voelkle M, Oud J, Davidov E, Schmidt P. An SEM approach to continuous time modeling of panel data: relating authoritarianism and anomia. Psychological Methods 2012; 17(2):176-192. 


\section{Statistics}

27. An X, Yang Q, Bentler P. A latent factor linear mixed model for high-dimensional longitudinal data analysis. Statistics in Medicine 2013; 32(24):4229-4239.

28. Zhang J, Stout W. The theoretical DETECT index of dimensionality and its application to approximate simple structure. Psychometrika 1999; 64(2):213-249.

29. Kaiser H. A second generation little jiffy. Psychometrika 1970; 35(4):401-415.

30. Lord F. Applications of Item Response Theory to Practical Testing Problems. L. Erlbaum Associates Hillsdale: NJ, 1980.

31. Samejima F. Estimation of latent ability using a response pattern of graded scores, 1969. Psychometrika Monographs No. 17.

32. Dunson D. Dynamic latent trait models for multidimensional longitudinal data. Journal of the American Statistical Association 2003; 98(463):555-563.

33. Duane S, Kennedy A, Pendleton B, Roweth D. Hybrid Monte Carlo. Physics Letters B 1987; 195(2):216-222.

34. Hoffman M, Gelman A. The no-U-turn sampler: adaptively setting path lengths in Hamiltonian Monte Carlo. The Journal of Machine Learning Research 2014; 15(1):1593-1623.

35. Stan Development Team. Stan modeling language users guide and reference manual, version 2.14.0, 2016. https://mc-stan. org/ [Accessed 1 May 2016].

36. Lunn D, Thomas A, Best N, Spiegelhalter D. WinBUGS - a Bayesian modelling framework: concepts, structure, and extensibility. Statistics and Computing 2000; 10(4):325-337.

37. Gelman A, Carlin J, Stern H, Dunson D, Vehtari A, Rubin D. Bayesian Data Analysis. CRC press: Boca Raton, 2013.

38. Celeux G, Forbes F, Robert C, Titterington D. Deviance information criteria for missing data models. Bayesian Analysis 2006; 1(4):651-673.

39. Cattell R. The scree test for the number of factors. Multivariate Behavioral Research 1966; 1(2):245-276.

40. Little R, Rubin D. Statistical Analysis with Missing Data. Wiley, John \& Sons: New York, 2002.

41. Diggle P, Heagerty P, Liang K, Zeger S. Analysis of Longitudinal Data. Oxford University Press: Oxford, 2002.

42. Millsap RE. Testing measurement invariance using item response theory in longitudinal data: an introduction. Child Development Perspectives 2010; 4(1):5-9.

43. McCulloch CE, Neuhaus JM. Misspecifying the shape of a random effects distribution: why getting it wrong may not matter. Statistical Science 2011; 26(3):388-402.

44. Escobar M. Estimating normal means with a Dirichlet process prior. Journal of the American Statistical Association 1994; 89(425):268-277. 